\title{
Search for single production of vector-like $B$ quark decaying to $b Z$ at future linear colliders
}

\author{
Lin Han ${ }^{a}$, Jie-Fen Shen ${ }^{\mathrm{b}}$ \\ School of Biomedical Engineering, Xinxiang Medical University, Xinxiang 453003, China
}

Received: 9 February 2021 / Accepted: 16 May 2021 / Published online: 27 May 2021

(C) The Author(s) 2021

\begin{abstract}
New vector-like quarks are predicted in many new physics scenarios beyond the Standard Model (SM). Based on a model-independent framework, we investigate the prospect of discovering the $S U$ (2) singlet vector-like bottom quark (VLQ- $B$ ) in $e^{+} e^{-}$collisions at $3 \mathrm{TeV}$ Compact Linear Collider. We study the single VLQ- $B$ production process $e^{-} e^{+} \rightarrow B \bar{b} \rightarrow Z b \bar{b}$ with two types of decay channel: $Z \rightarrow \ell^{+} \ell^{-}$, and $Z \rightarrow v \bar{v}$. By carrying out a full simulation for the signals and the relevant SM backgrounds, the $2 \sigma$ exclusion limit and $5 \sigma$ discovery prospects are, respectively, obtained on the $B$ quark mass and the coupling strength $g^{*}$ with the integrated luminosity of $5 \mathrm{ab}^{-1}$. Our numerical results show that the possible signals of the singlet VLQ$B$ might be detected at the future high-energy $e^{+} e^{-}$linear colliders.
\end{abstract}

\section{Introduction}

The discovery of the Higgs boson by the ATLAS and CMS Collaborations at the Large Hadron Collider (LHC) [1,2] opened a new field for explorations in the realm of particle physics. To solve the gauge hierarchy problem, the vectorlike quarks (VLQs) are predicted to regulate the Higgs boson mass-squared divergence [3] in many new physics (NP) scenarios beyond the Standard Model (SM), such as little Higgs models [4], extra dimensions [5], composite Higgs models [6], and other extended models [7-10]. These new particles are color-triplet spin-1/2 fermions and the left- and right-handed components transform with the same properties under the SM electroweak symmetry group [11].

The electric charges of VLQs could be $+2 / 3 e$ ( $T$ quark), $-1 / 3 e$ ( $B$ quark), $+5 / 3 e$ ( $X$ quark or $-4 / 3 e$ ( $Y$ quark), and they could be appear in electroweak singlet $[T, B]$,

\footnotetext{
a e-mail: hanlin@xxmu.edu.cn

be-mail: shjf@xxmu.edu.cn (corresponding author)
}

electroweak doublets $[(X, T),(T, B)$, or $(B, Y)]$, or electroweak triplets $[(X, T, B)$ or $(T, B, Y)]$. A common feature of these new fermions is that they could decay into a SM quark and a SM gauge boson, or a Higgs boson, which might generate characteristic signatures at the current and future high-energy colliders (for example see [12-42]). Here we focus on the singlet VLQ- $B$ quark, which couples exclusively to third-generation SM quarks, $B \rightarrow W t, H b, Z b$.

Up to now, the direct searches for VLQ- $B$ have been performed by the ATLAS and CMS Collaborations in Run 2 [43-51]. Although there is no experimental evidence of such VLQ- $B$, the constraints on VLQ- $B$ have been obtained at 95\% condifence level (CL). For instance, an analysis from CMS including single-lepton, dilepton, and multilepton final states probed all decay modes of the VLQ- $B$, and excluded their masses in the range 910-1240 GeV [49], depending on the combination of the VLQ branching fractions. Very recently, the CMS Collaboration presented a search for VLQ$B$ pair production in the fully hadronic final state using Run 2 data with a total integrated luminosity $137 \mathrm{fb}^{-1}$ [50], and excluded their masses up to 1570,1390 , and $1450 \mathrm{GeV}$ for $100 \% B \rightarrow b h, 100 \% B \rightarrow t Z$, and $B Y$ doublet cases, respectively. In addition, a combination of searches utilizing various final states were performed by the ATLAS Collaboration [51], and excluded values of the VLQ- $B$ mass up to 1220,1370 , and $1140 \mathrm{GeV}$ for the singlet, the $(T, B)$ doublet and $(B, Y)$ doublet cases, respectively.

So far, most of the work about the VLQ- $B$ focuses on phenomenological analysis at the hadron colliders [52-54]. Compared with the hadron colliders, a future linear $e^{+} e^{-}$collider has a particularly clear background environment [5557], i.e., the final stage of Compact Linear Collider (CLIC) operating at an energy of $3 \mathrm{TeV}$ is expected to directly examine the pair production of new heavy top partner of mass up to $1.5 \mathrm{TeV}$ [58]. The high-energy linear collider is thus a precision machine with which the properties of new VLQs can be measured precisely [59-65]. To the best of our knowledge, no 

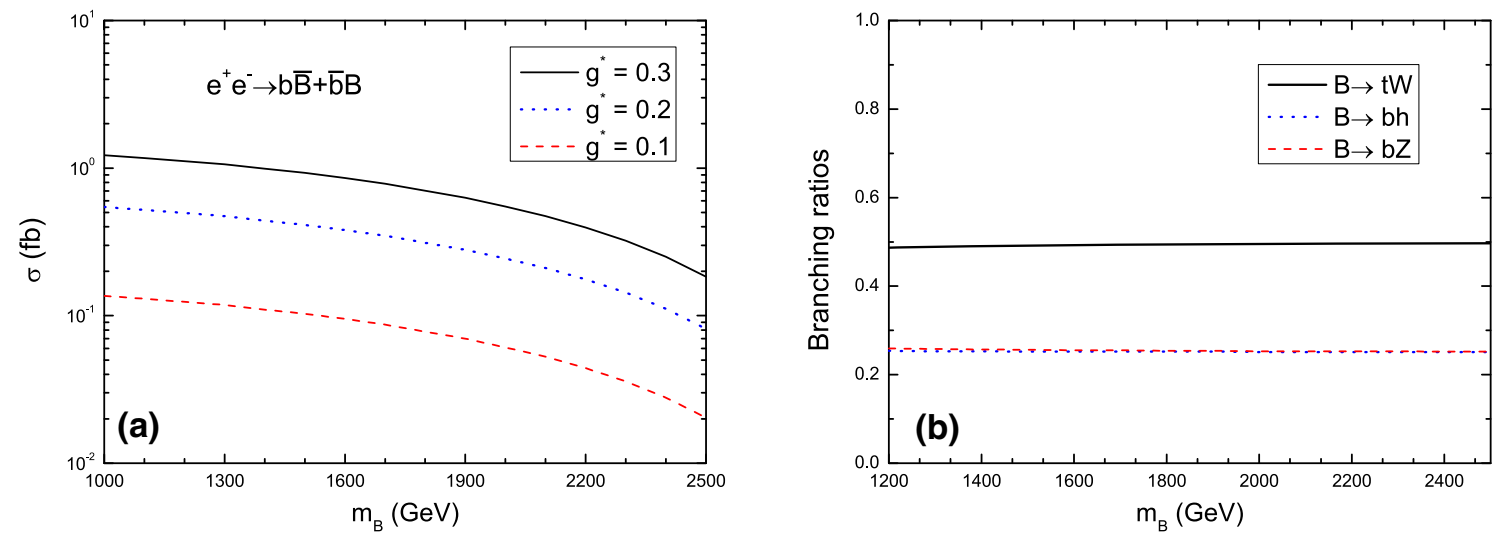

Fig. 1 a Total cross sections as a function of $m_{B}$ with three typical values of $g^{*}$. b Branching ratios for the three decay modes for various of VLQ- $B$ quark masses with $g^{*}=0.1$

work has been done to search single production of the $S U$ (2) singlet VLQ- $B$ in $e^{+} e^{-}$collisions. Therefore, we focus on the observability of the single VLQ- $B$ production at the CLIC via the process $e^{+} e^{-} \rightarrow B \bar{b}$ combined with the $B \rightarrow b Z$ and the subsequent decay channels $Z \rightarrow \ell^{+} \ell^{-}$, and $Z \rightarrow v \bar{v}$, respectively. The benefit is that it is a more highly potential process than pair production owing to less phase-space suppression. We expect that such work may become a complementary option to other production processes in searches for the heavy VLQ- $B$ at the future high-energy linear colliders.

This paper is organized as follows: in Sect. 2, we brief review the couplings of VLQ- $B$ with the SM particles, and discuss its single production at future $3 \mathrm{TeV}$ CLIC and the possible decay modes. Section 3 is devoted to a detailed analysis of the relevant signals and backgrounds at the CLIC. Finally, we summarize our results in Sect. 4.

\section{Singlet vector-like bottom quark in the simplified model}

Following the notation of Ref. [14], a simple Lagrangian that parametrizes the VLQ- $B$ couplings to quarks and electroweak boson can be expressed as (showing only the couplings relevant for our analysis)

$$
\begin{aligned}
\mathcal{L}= & \frac{g g^{*}}{2}\left[\frac{1}{\sqrt{2}}\left[\bar{B}_{L} W_{\mu}^{-} \gamma^{\mu} t_{L}\right]+\frac{1}{2 \cos \theta_{W}}\left[\bar{B}_{L} Z_{\mu} \gamma^{\mu} b_{L}\right]\right. \\
& \left.-\frac{M_{B}}{2 m_{W}}\left[\bar{B}_{R} H b_{L}\right]-\frac{m_{b}}{2 m_{W}}\left[\bar{B}_{L} H b_{R}\right]\right]+ \text { h.c. }
\end{aligned}
$$

where $g$ is the $S U(2)_{L}$ gauge coupling constant and $\theta_{W}$ is the Weinberg angle. Thus there are only two model parameters: the VLQ- $B$ quark mass $m_{B}$ and the coupling strength to SM quarks in units of standard couplings, $g^{*}$.
In order to make a prediction for the signal, we calculate the cross section for the process $e^{+} e^{-} \rightarrow B \bar{b}+b \bar{B}$ at leading order (LO) by using MadGraph5-aMC@NLO [66]. Note that the model file of the singlet VLQ- $B$ quark could be downloaded from the Feynrules Model Database [67] and could also be implemented via the FeynRules package [68]. The numerical values of the input parameters are taken from [69].

In Fig. 1a, we show the dependence of the cross sections for the process $e^{+} e^{-} \rightarrow B \bar{b}+b \bar{B}$ on the VLQ- $B$ quark mass $m_{B}$ at a $3 \mathrm{TeV}$ CLIC for three typical values of $g^{*}$. As the VLQ- $B$ quark mass grows, the cross section of single production decreases slowly due to a larger phase space. For $g^{*}=0.2$ and $m_{B}=1.5$ (2) $\mathrm{TeV}$, the cross section can reach $0.41(0.24) \mathrm{fb}$. Obviously, the cross section of single $B$-quark production is proportional to the square of the coupling strength $g^{*}$ for a given VLQ- $B$ quark mass. In Fig. 1b, we also show the branching ratios (BRs) of the three decay channels $B \rightarrow t W, b Z$, and $b h$ by varying the $B$ quark mass at fixed $g^{*}=0.1$. One can see that $\mathrm{BR}(B \rightarrow b Z) \approx \mathrm{BR}(B \rightarrow b h) \approx \frac{1}{2} \mathrm{BR}(B \rightarrow W t)$ is a good approximation, as expected from the Goldstone boson equivalence theorem [70-74].

\section{Collider simulation and analysis}

From the above discussions, we know that the VLQ- $B$ can be singly produced through $s$-channel $Z$ boson exchange by $e^{+} e^{-}$collisions. The relevant Feynman diagrams for single production and decaying into $b Z$ are depicted in Fig. 2.

Considering the subsequent leptonic decay channel $Z \rightarrow$ $\ell^{+} \ell^{-}$, and invisible decay channel $Z \rightarrow v \bar{v}$, there are two typical final states, $2 b 2 \ell$, and $2 b+\mathbb{E}_{T}$, respectively.

Monte Carlo event simulations for signal and SM background are interfaced to Pythia 8.20 [75] for fragmentation 


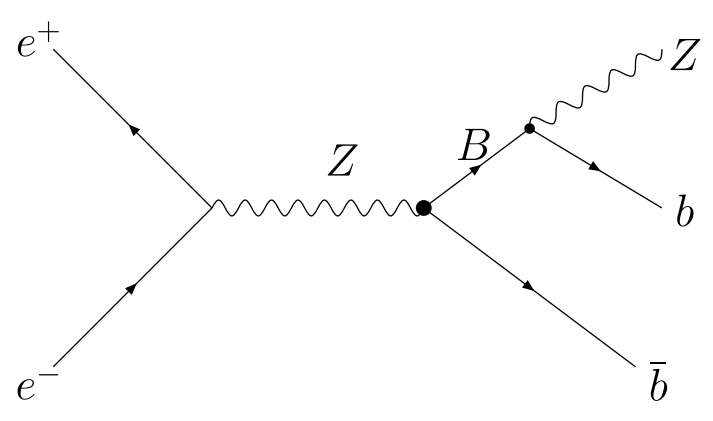

Fig. 2 Representative Feynman diagrams of the processes $e^{+} e^{-} \rightarrow$ $B(\rightarrow Z b) \bar{b}$

and showering. All event samples are fed into the Delphes 3.4.2 [76] with the CLIC detector card designed for $3 \mathrm{TeV}$ [77]. In our analysis, jets are clustered with the Valencia Linear Collider (VLC) algorithm $[78,79]$ in exclusive mode with a fixed number of jet ( $N=2$ where $N$ corresponds to the number of partons expected in the tell level final state) and fixed one size parameter $R=1.0$. The $b$-tagging efficiency and misidentification rates are taken as the medium working points (WP) (70\% b-tagging efficiency) and the misidentification rates are given as a function of energy and pseudorapidity, i.e., in a bit where $E>500 \mathrm{GeV}$ and $1.53<|\eta| \leq 2.09$, misidentification rates are $9 \times 10^{-3}$ for the medium WP. Finally, event analysis is performed by using MadAnalysis5 [80].

\subsection{Analysis of decay channel $Z \rightarrow \ell^{+} \ell^{-}$}

In this subsection, we analyze the signal and background events at $3 \mathrm{TeV}$ CLIC through the $Z \rightarrow \ell^{+} \ell^{-}(\ell=e, \mu)$ decay channel.

$e^{+} e^{-} \rightarrow B \bar{b} \rightarrow Z b \bar{b} \rightarrow \ell^{+} \ell^{-} b \bar{b}$.

For this channel, the typical signal is two $b$-jet and two Opposite-Sign and Same-Flavor (OSSF) leptons. The main SM backgrounds come from the following processes:

- $e^{+} e^{-} \rightarrow b \bar{b} \ell^{+} \ell^{-}$

- $e^{+} e^{-} \rightarrow j j \ell^{+} \ell^{-}$,

- $e^{+} e^{-} \rightarrow t \bar{t} \rightarrow b \bar{b} \ell^{+} \ell^{-}+\mathbb{E}_{T}$,

- $e^{+} e^{-} \rightarrow Z W^{+} W^{-} \rightarrow b \bar{b} \ell^{+} \ell^{-}+\mathbb{E}_{T}$.

To identify objects, we choose the basic cuts at parton level for the signals and SM backgrounds as follows:

$p_{T}^{\ell}>15 \mathrm{GeV}, \quad p_{T}^{j / b}>25 \mathrm{GeV}, \quad\left|\eta_{\ell / b / j}\right|<2.5$

where $\Delta R=\sqrt{\Delta \Phi^{2}+\Delta \eta^{2}}$ denotes the separation in the rapidity-azimuth plane, $p_{T}^{\ell, b, j}$ are the transverse momentum of leptons, $b$-jets, and light jets.
For the signal, the leptons $\ell_{1}$ and $\ell_{2}$ are two OSSF leptons that are assumed to be the product of the $Z$-boson decay, and at least two $b$-tagged jet are present. In Fig. 3, we plot some differential distributions for signals and SM backgrounds at the CLIC, such as the transverse momentum distributions of the leading and sub-leading $b$-jets $\left(p_{T}^{b_{1}}, p_{T}^{b_{2}}\right)$, the separations $\Delta R_{b_{1}, b_{2}}$, the transverse momentum distributions of the leading and sub-leading leptons $\left(p_{T}^{\ell_{1} \ell_{2}}\right.$ ), the separations $\Delta R_{\ell_{1}, \ell_{2}}$, and the invariant mass distribution for the $B$ quark $M_{b \ell_{1} \ell_{2}}$. Based on these kinematical distributions, we can impose the following set of cuts:

- Cut-1: There are exactly two isolated leptons $(N(\ell)=2)$ and two $b$-tagged jets $(N(b)=2)$, and the transverse momenta of the leading and sub-leading $b$-jet are required $p_{T}^{b_{1}}>400 \mathrm{GeV}$ and $p_{T}^{b_{2}}>200 \mathrm{GeV}$ with $\Delta R_{b_{1}, b_{2}}>$ 2.5 . Besides, the transverse momenta of two leptons from the $Z$ boson are required to have $p_{T}^{\ell_{1} \ell_{2}}>200 \mathrm{GeV}$ with $\Delta R_{\ell_{1}, \ell_{2}}<1$.

- Cut-2: The invariant mass of the $Z$ boson is required to have $\left|M_{\ell_{1} \ell_{2}}-m_{Z}\right|<10 \mathrm{GeV}$.

- Cut-3: The invariant mass the VLQ- $B$ quark is required to have $M_{b_{1} \ell_{1} \ell_{2}}>1000 \mathrm{GeV}$.

We present the cross sections of three typical signal $\left(m_{B}=\right.$ $1200,1500,2000 \mathrm{GeV}$ ) and the relevant backgrounds after imposing the cuts in Table 1. One can see that all the SM backgrounds are suppressed very efficiently, while the signals still have a relatively good efficiency at the end of the cut flow. The large background comes from the $e^{+} e^{-} \rightarrow b \bar{b} \ell^{+} \ell^{-}$ process, with a total cross section of $1.92 \mathrm{ab}$.

\subsection{Analysis of invisible decay channels $Z \rightarrow v \bar{v}$}

In this subsection, we analyze the signal and background events through the invisible decays $Z \rightarrow v \bar{v}$ decay channel.

$e^{+} e^{-} \rightarrow B \bar{b} \rightarrow Z b \bar{b} \rightarrow b \bar{b}+\mathbb{E}_{T}$.

For this channel, the main SM backgrounds come from the following processes:

- $e^{+} e^{-} \rightarrow v \bar{v} b \bar{b}$,

- $e^{+} e^{-} \rightarrow v \bar{v} j j$.

In our simulations, we apply the following basic cuts on the signal and background events:

$p_{T}^{b / j}>30 \mathrm{GeV}, \quad\left|\eta_{b / j}\right|<2.5, \quad \mathbb{E}_{T}>100 \mathrm{GeV}$.

In order to get some hints of further cuts for reducing the SM backgrounds, we analyzed the normalized distributions of the missing transverse energy $\mathbb{E}_{T}, p_{T}^{b_{1}}, p_{T}^{b_{2}}, \Delta R_{b_{1}, b_{2}}$, and the transverse mass distribution for the $\mathrm{B}$ quark $M_{T}\left(b_{1}\right)$ for 

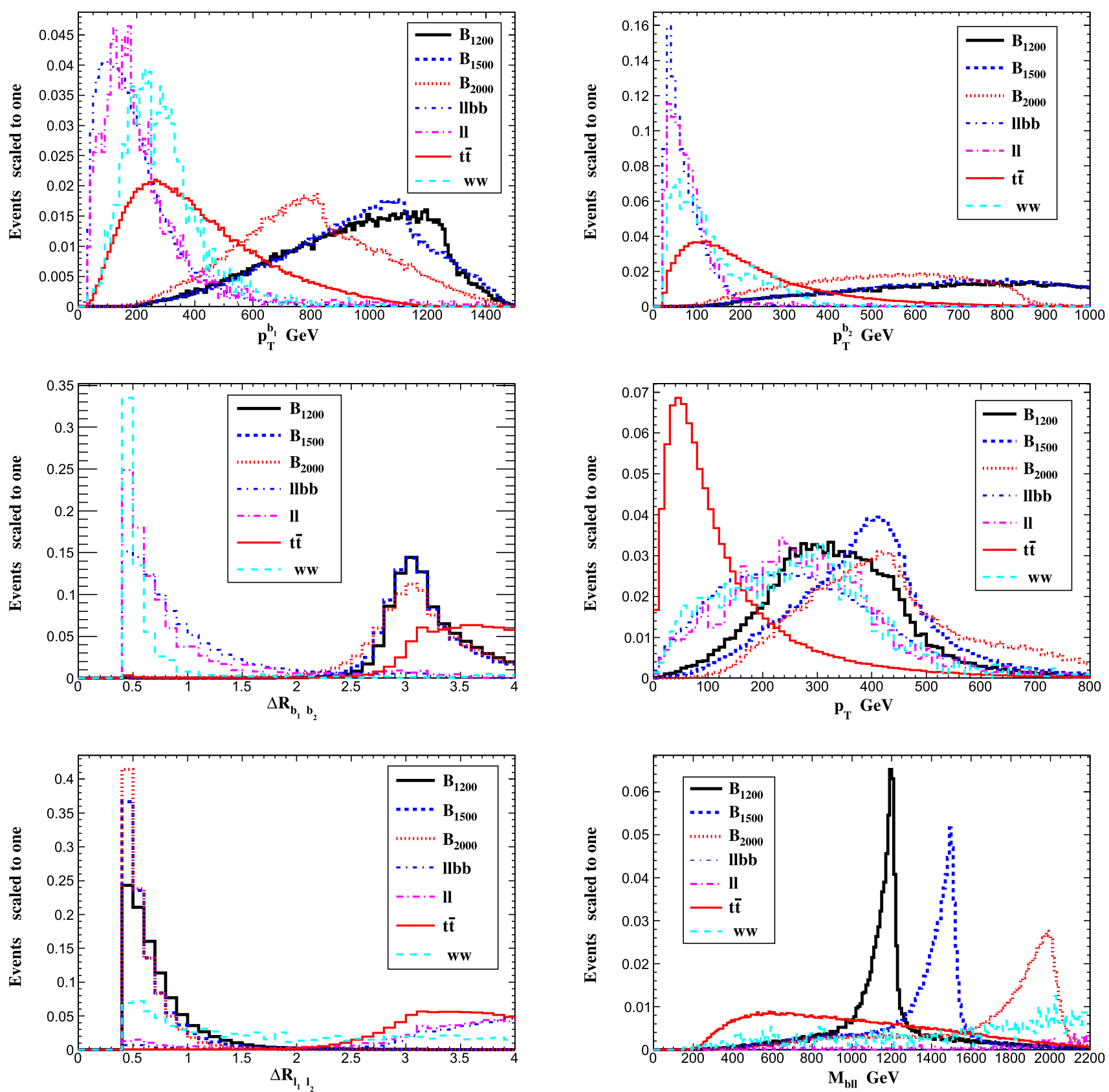

Fig. 3 Normalized distributions for the signals (with $m_{B}=1200,1500$ and $2000 \mathrm{GeV}$ ) and SM backgrounds at the CLIC

Table 1 Cut flow of the cross sections (in ab) for the signals and SM backgrounds at the 3 TeV CLIC with $g^{*}=0.1$ and three typical VLQ- $B$ quark masses

\begin{tabular}{|c|c|c|c|c|c|c|c|}
\hline \multirow[t]{2}{*}{ Cuts } & \multicolumn{3}{|l|}{ Signals } & \multicolumn{4}{|c|}{ Backgrounds } \\
\hline & $\overline{1200 \mathrm{GeV}}$ & $1500 \mathrm{GeV}$ & $2000 \mathrm{GeV}$ & $\overline{\ell^{+}} \ell^{-} b b$ & $\ell^{+} \ell^{-} j j$ & $t \bar{t}$ & $Z W^{+} W^{-}$ \\
\hline Basic & 1.02 & 0.62 & 0.16 & 3627 & 10,480 & 646 & 173 \\
\hline Cut 1 & 0.39 & 0.23 & 0.052 & 2.84 & 0.05 & 0.004 & 0.0021 \\
\hline Cut 2 & 0.30 & 0.2 & 0.045 & 2.48 & 0.043 & 0.0006 & 0.002 \\
\hline Cut3 & 0.27 & 0.16 & 0.04 & 1.92 & 0.027 & 0.0004 & 0.0012 \\
\hline
\end{tabular}



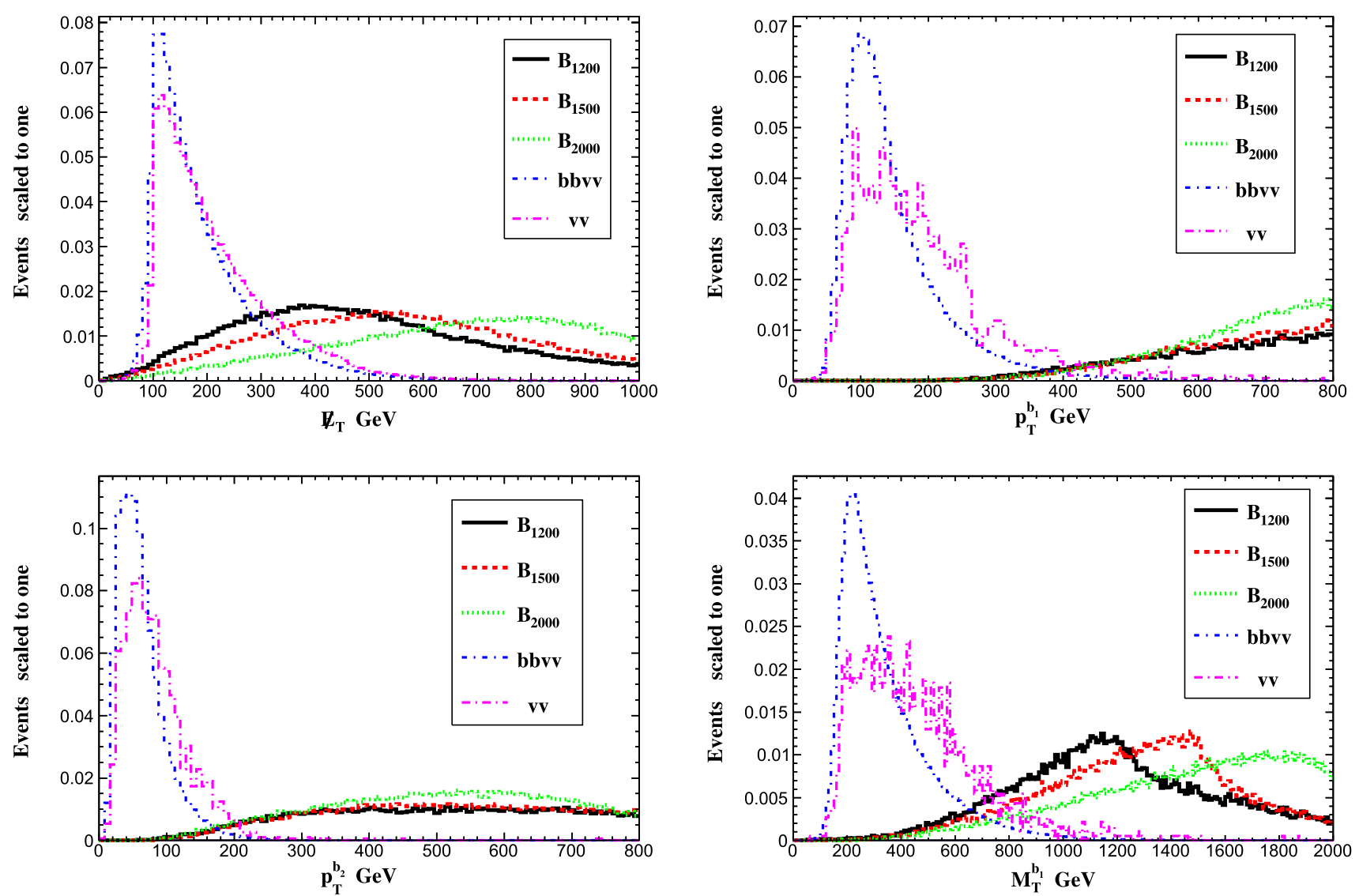

Fig. 4 Normalized distributions for the signals (with $m_{B}=1200,1500$ and $2000 \mathrm{GeV}$ ) and SM backgrounds at the CLIC

signals and SM backgrounds as shown in Fig. 4. Due to the existence of neutrinos in the final state of signal events, the $B$ candidate transverse mass is reconstructed as $M_{T}\left(b_{1}\right)=$ $\sqrt{2 p_{T, b_{1}} E_{T}^{m i s s}-\mathbf{p}_{T, b_{1}} \cdot \mathbf{E}_{T}^{\text {miss }}}$. Note that the definition of the transverse mass of the system can be seen from Ref. [81].

Based on these kinematical distributions, a set of further cuts are given as follows:

- Cut-1: The transverse missing energy is required $\mathbb{E}_{T}>$ $300 \mathrm{GeV}$.

- Cut-2: There are at least two $b$-tagged jets $(N(b) \geq 2)$ and remove any electrons and muons $(N(\ell)=0)$, and the transverse momenta of the leading and sub-leading $b$-jet are required $p_{T}^{b_{1}}>400 \mathrm{GeV}$ and $p_{T}^{b_{2}}>200 \mathrm{GeV}$ with $\Delta R_{b_{1}, b_{2}}>2.5$.

- Cut-3: The transverse mass of the $B$ quark, $M_{T}\left(b_{1}\right)>$ $1000 \mathrm{GeV}$.

We summarize the cross sections of three typical signal $\left(m_{B}=1200,1500,2000 \mathrm{GeV}\right)$ and the relevant backgrounds after imposing the cuts in Table 2. One can see that the large background comes from the $e^{+} e^{-} \rightarrow v \bar{v} b \bar{b}$ process, with a total cross section of $17.4 \mathrm{ab}$.

\subsection{Discovery and exclusion significance}

To handle the relatively small event number, we will use the median significance $\mathcal{Z}$ to estimate the expected discovery and exclusion significance [82]:

$$
\begin{array}{r}
\mathcal{Z}_{\mathrm{disc}}=\sqrt{2[(s+b) \ln (1+s / b)-s]} \geq 5, \\
\mathcal{Z}_{\mathrm{excl}}=\sqrt{2[s-b \ln (1+s / b)]} \leq 2 .
\end{array}
$$

Here, $s$ and $b$ stand for the numbers of signal and SM background events after cuts in which the integrated luminosity are taken as $5 \mathrm{ab}^{-1}$ at $3 \mathrm{TeV}$ CLIC.

In Fig. 5, we plot the $2 \sigma$ and $5 \sigma$ sensitivity reaches for the coupling strength $g^{*}$ as a function of $m_{B}$ at $3 \mathrm{TeV}$ CLIC with integral luminosity $5 \mathrm{ab}^{-1}$. One finds that, for the $Z \rightarrow \ell^{+} \ell^{-}$ decay channel, the VLQ- $B$ quark can be excluded in the region of $g^{*} \in[0.25,0.5]$ and $m_{B} \in[1200 \mathrm{GeV}, 1850 \mathrm{GeV}]$ at the $3 \mathrm{TeV}$ CLIC with the integrated luminosity of $5 \mathrm{ab}^{-1}$, 
Table 2 Cut flow of the cross sections (in ab) for the signals and SM backgrounds at the CLIC with $g^{*}=0.1$ and three typical VLQ- $B$ quark masses

\begin{tabular}{|c|c|c|c|c|c|}
\hline \multirow[t]{2}{*}{ Cuts } & \multicolumn{3}{|l|}{ Signals } & \multicolumn{2}{|c|}{ Backgrounds } \\
\hline & $1200 \mathrm{GeV}$ & $1500 \mathrm{GeV}$ & $2000 \mathrm{GeV}$ & $\overline{v \bar{v} b b}$ & $\nu \bar{v} j j$ \\
\hline Basic & 5.92 & 4.89 & 2.85 & 281,000 & 414,300 \\
\hline Cut 1 & 4.16 & 3.78 & 2.39 & 31753 & 1533 \\
\hline Cut 2 & 1.56 & 1.27 & 0.62 & 28 & 0.12 \\
\hline Cut3 & 1.36 & 1.12 & 0.57 & 17.4 & 0.08 \\
\hline
\end{tabular}
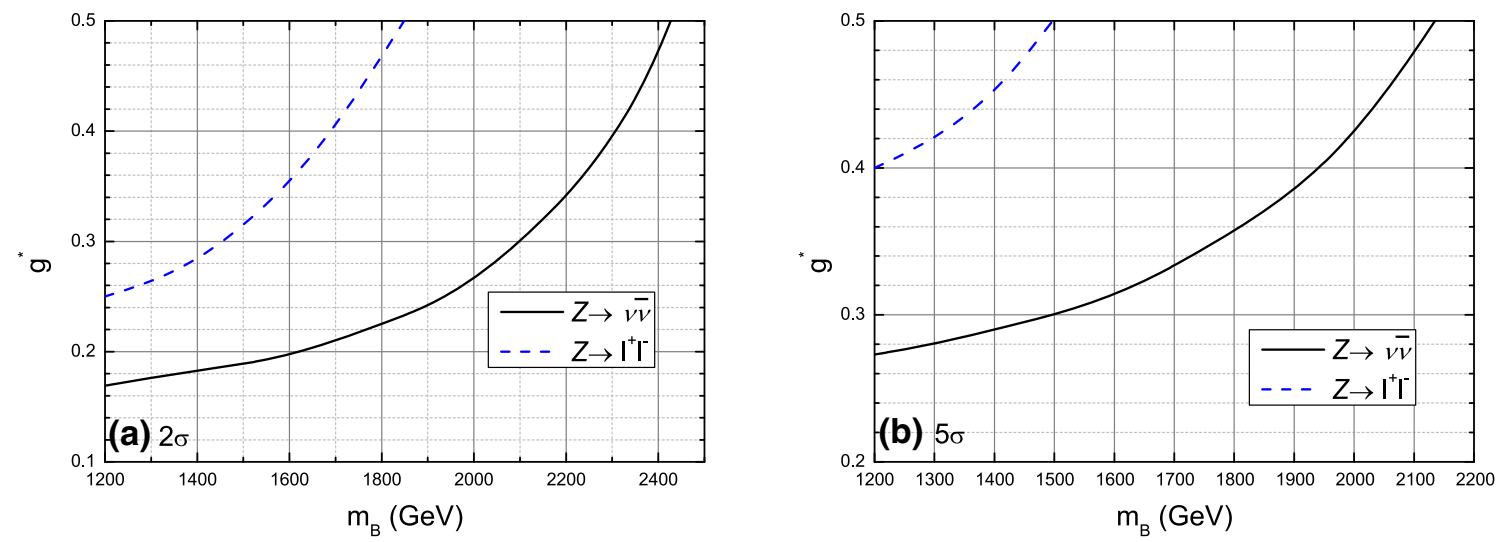

Fig. $52 \sigma$ exclusion limit (left) and $5 \sigma$ discovery prospects (right) contour plots for the signal in $g^{*}-m_{B}$ planes at 3 TeV CLIC with integral luminosity $5 \mathrm{ab}^{-1}$

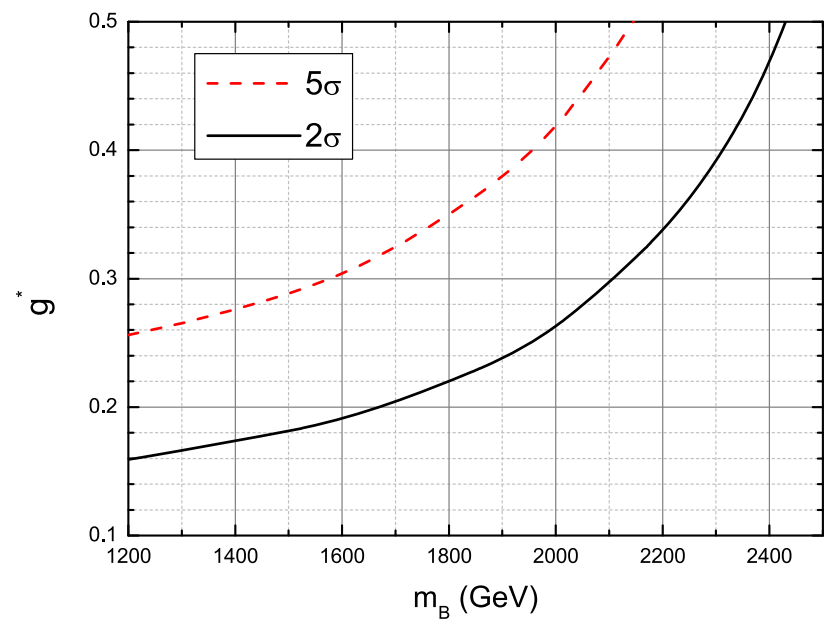

Fig. 6 Combined $2 \sigma$ exclusion limit and $5 \sigma$ discovery prospect contour plots for the signal in $g^{*}-m_{B}$ planes at CLIC with integral luminosity $5 \mathrm{ab}^{-1}$

while the discovery region can reach $g^{*} \in[0.4,0.5]$ and $m_{B} \in[1200 \mathrm{GeV}, 1500 \mathrm{GeV}]$. While for $Z \rightarrow v \bar{v}$ decay channel, the VLQ- $B$ quark mass can be excluded in the region of $g^{*} \in[0.17,0.5]$ and $m_{B} \in[1200 \mathrm{GeV}, 2400 \mathrm{GeV}]$ and the discovery region can reach $g^{*} \in[0.27,0.5]$ and $m_{B} \in$ [1200 GeV, $2150 \mathrm{GeV}]$.
Then, we combine its sensitivity with the above two types of decay channels by using $\mathcal{Z}_{\text {comb }}=\sqrt{\mathcal{Z}_{\ell \bar{\ell}}^{2}+\mathcal{Z}_{v \bar{v}}^{2}}$. For comparison, we further present in Fig. 6 the combined sensitivity reaches for the coupling strength $g^{*}$ as a function of the VLQ- $B$ quark mass $m_{B}$. One can see that the $B$ quark can be excluded in the region of $g^{*} \in[0.16,0.5]$ and $m_{B} \in$ $[1200 \mathrm{GeV}, 2400 \mathrm{GeV}$ ] at the $3 \mathrm{TeV}$ CLIC with the integrated luminosity of $5 \mathrm{ab}^{-1}$, while the discovery region can reach $g^{*} \in[0.26,0.5]$ and $m_{B} \in[1200 \mathrm{GeV}, 2150 \mathrm{GeV}]$. From above discussions, we can see that, for single production of VLQ- $B$ with the subsequent $B \rightarrow b Z$ decay channel at 3 $\mathrm{TeV}$ CLIC, it is possible to detect its signal via $2 b 2 \ell$ and $2 b+\mathbb{E}_{T}$ final states, respectively.

\section{Conclusion}

In this work, we analyzed single production of the $\mathrm{SU}(2)$ singlet VLQ- $B$ at the future $3 \mathrm{TeV}$ CLIC via the process $e^{+} e^{-} \rightarrow B \bar{b} \rightarrow Z b \bar{b}$ in a simplified model, in which only two free parameters are included, the VLQ- $B$ quark mass $m_{B}$ and the EW coupling constant $g^{*}$. We performed a full simulation for the signals and the relevant SM backgrounds based on two types of decay channels $Z \rightarrow \ell^{+} \ell^{-}(\ell=e, \mu)$ and $Z \rightarrow v \bar{v}$. The $5 \sigma$ discovery prospects and $2 \sigma$ exclusion limits in the parameter plane of the two variables $m_{B}$ and $g^{*}$ 
were obtained at $3 \mathrm{TeV}$ CLIC with the integral luminosity of $5 \mathrm{ab}^{-1}$, including the combined reaches of both leptonic and invisible channels. Our numerical results show that the VLQ- $B$ can be excluded in the region of $g^{*} \in[0.16,0.5]$ and $m_{B} \in[1200 \mathrm{GeV}, 2400 \mathrm{GeV}]$ and the discovery region can reach $g^{*} \in[0.26,0.5]$ and $m_{B} \in[1200 \mathrm{GeV}, 2150 \mathrm{GeV}]$. We therefore expect that the signatures studied here will provide complementary information for detecting such singlet VLQ$B$ quark in the future $3 \mathrm{TeV}$ CLIC.

Acknowledgements We thank Yao-Bei Liu for helpful discussion. The work is supported by the Foundation of the Xinxiang Medical University (Grant no. 505035).

Data Availability Statement This manuscript has no associated data or the data will not be deposited. [Authors' comment: All data has been included in the table and figures.]

Open Access This article is licensed under a Creative Commons Attribution 4.0 International License, which permits use, sharing, adaptation, distribution and reproduction in any medium or format, as long as you give appropriate credit to the original author(s) and the source, provide a link to the Creative Commons licence, and indicate if changes were made. The images or other third party material in this article are included in the article's Creative Commons licence, unless indicated otherwise in a credit line to the material. If material is not included in the article's Creative Commons licence and your intended use is not permitted by statutory regulation or exceeds the permitted use, you will need to obtain permission directly from the copyright holder. To view a copy of this licence, visit http://creativecomm ons.org/licenses/by/4.0/.

Funded by SCOAP ${ }^{3}$.

\section{References}

1. G. Aad et al., ATLAS Collaboration. Phys. Lett. B 716, 1 (2012)

2. S. Chatrchyan et al., CMS Collaboration. Phys. Lett. B 716, 30 (2012)

3. A. De Simone, O. Matsedonskyi, R. Rattazzi, A. Wulzer, JHEP 04, 004 (2013)

4. N. Arkani-Hamed, A.G. Cohen, E. Katz, A.E. Nelson, JHEP 0207, 034 (2002)

5. K. Agashe, G. Perez, A. Soni, Phys. Rev. D 75, 015002 (2007)

6. K. Agashe, R. Contino, A. Pomarol, Nucl. Phys. B 719, 165 (2005)

7. H.J. He, T.M.P. Tait, C.P. Yuan, Phys. Rev. D 62, 011702 (2000)

8. X.F. Wang, C. Du, H.J. He, Phys. Lett. B 723, 314-323 (2013)

9. H.J. He, C.T. Hill, T.M.P. Tait, Phys. Rev. D 65, 055006 (2002)

10. H.J. He, Z.Z. Xianyu, JCAP 10, 019 (2014)

11. J.A. Aguilar-Saavedra, R. Benbrik, S. Heinemeyer, M. PrezVictoria, Phys. Rev. D 88, 094010 (2013)

12. J.A. Aguilar-Saavedra, JHEP 0911, 030 (2009)

13. A. Atre, G. Azuelos, M. Carena, T. Han, E. Ozcan, J. Santiago, G. Unel, JHEP 1108, 080 (2011)

14. M. Buchkremer, G. Cacciapaglia, A. Deandrea, L. Panizzi, Nucl. Phys. B 876, 376 (2013)

15. A. Atre, M. Carena, T. Han, J. Santiago, Phys. Rev. D 79, 054018 (2009)

16. D. Barducci, A. Belyaev, J. Blamey, S. Moretti, L. Panizzi, H. Prager, JHEP 1407, 142 (2014)

17. S.A.R. Ellis, R.M. Godbole, S. Gopalakrishna, J.D. Wells, JHEP 1409, 130 (2014)
18. D. Barducci et al., JHEP 1412, 080 (2014)

19. M. Backović, T. Flacke, S.J. Lee, G. Perez, JHEP 1509, 022 (2015)

20. M. Backovic, T. Flacke, J.H. Kim, S.J. Lee, JHEP 1604, 014 (2016)

21. D. Barducci, L. Panizzi, JHEP 1712, 057 (2017)

22. G. Cacciapaglia, A. Carvalho, A. Deandrea, T. Flacke, B. Fuks, D. Majumder, L. Panizzi, H.S. Shao, Phys. Lett. B 793, 206-211 (2019)

23. B. Fuks, H.S. Shao, Eur. Phys. J. C 77, 135 (2017)

24. S. Yang, J. Jiang, Q.S. Yan, X. Zhao, JHEP 1409, 035 (2014)

25. H.J. He, C.T. Hill, T.M.P. Tait, Phys. Rev. D 65, 055006 (2002)

26. H.J. He, T.M.P. Tait, C.P. Yuan, Phys. Rev. D 62, 011702 (2000)

27. X.F. Wang, C. Du, H.J. He, Phys. Lett. B 723, 314 (2013)

28. H.J. He, Z.Z. Xianyu, JCAP 1410, 019 (2014)

29. D. Liu, L.T. Wang, K.P. Xie, JHEP 1901, 157 (2019)

30. G. Cacciapaglia, A. Deandrea, N. Gaur, D. Harada, Y. Okada, L. Panizzi, JHEP 11, 055 (2018)

31. Y.J. Zhang, L. Han, Y.B. Liu, Phys. Lett. B 768, 241 (2017)

32. L. Han, Y.J. Zhang, Y.B. Liu, Phys. Lett. B 771, 106 (2017)

33. Y.B. Liu, Phys. Rev. D 95, 035013 (2017)

34. Y.B. Liu, Y.Q. Li, Eur. Phys. J. C 77, 654 (2017)

35. Y.B. Liu, S. Moretti, Phys. Rev. D 100, 015025 (2019)

36. M. Chala, Phys. Rev. D 96, 015028 (2017)

37. J.A. Aguilar-Saavedra, J. Alonso-González, L. Merlo, J.M. No, Phys. Rev. D 101, 035015 (2020)

38. D. Wang, L. Wu, M. Zhang, arXiv:2007.09722 [hep-ph]

39. S. Moretti, D. O'Brien, L. Panizzi, H. Prager, Phys. Rev. D 96, 075035 (2017)

40. A. Carvalho, S. Moretti, D. O'Brien, L. Panizzi, H. Prager, Phys. Rev. D 98, 015029 (2018)

41. A. Roy, N. Nikiforou, N. Castro, T. Andeen, Phys. Rev. D 101, $115027(2020)$

42. A. Buckley, J.M. Butterworth, L. Corpe, D. Huang, P. Sun, Sci. Post Phys. 9, 069 (2020)

43. M. Aaboud et al., ATLAS Collaboration, Phys. Rev. D 98, 092005 (2018)

44. M. Aaboud et al., ATLAS Collaboration, JHEP 1812, 039 (2018)

45. M. Aaboud et al., ATLAS Collaboration, JHEP 1808, 048 (2018)

46. M. Aaboud et al., ATLAS Collaboration, JHEP 1905, 164 (2019)

47. A.M. Sirunyan et al., CMS Collaboration, Eur. Phys. J. C 79, 364 (2019)

48. A.M. Sirunyan et al., CMS Collaboration, Phys. Rev. D 100, 072001 (2019)

49. A.M. Sirunyan et al., CMS Collaboration, JHEP 08, 177 (2018)

50. A.M. Sirunyan et al., CMS Collaboration, Phys. Rev. D 102, $112004(2020)$

51. M. Aaboud et al., ATLAS Collaboration, Phys. Rev. Lett. 121, 211801 (2018)

52. J. Nutter, R. Schwienhorst, D.G.E. Walker, J.H. Yu, Phys. Rev. D 86, 094006 (2012)

53. X. Gong, C.X. Yue, Y.C. Guo, Phys. Lett. B 793, 175 (2019)

54. X. Gong, C.X. Yue, H.M. Yu, D. Li, Eur. Phys. J. C 80, 876 (2020)

55. H. Abramowicz et al. [CLIC Detector and Physics Study], arXiv: 1307.5288 [hep-ex]

56. J. de Blas, R. Franceschini, F. Riva, P. Roloff, U. Schnoor, M. Spannowsky, J.D. Wells, A. Wulzer, J. Zupan, S. Alipour-Fard et al., arXiv:1812.02093 [hep-ph]

57. R. Franceschini, Int. J. Mod. Phys. A 35, 2041015 (2020)

58. D. Dannheim, P. Lebrun, L. Linssen, D. Schulte, S. Stapnes, arXiv:1305.5766 [physics.acc-ph]

59. R. Kitano, T. Moroi, S.F. Su, JHEP 12, 011 (2002)

60. K. Kong, S.C. Park, JHEP 08, 038 (2007)

61. A. Senol, A.T. Tasci, F. Ustabas, Nucl. Phys. B 851, 289-297 (2011)

62. K. Harigaya, S. Matsumoto, M.M. Nojiri, K. Tobioka, JHEP 01, 135 (2012)

63. L. Guo, W. Liu, W.G. Ma, R.Y. Zhang, W.J. Zhang, Commun. Theor. Phys. 62(6), 824-832 (2014) 
64. Y.B. Liu, Z.J. Xiao, Nucl. Phys. B 892, 63-82 (2015)

65. X. Qin, J.-F. Shen, Nucl. Phys. B 966, 115388 (2021)

66. J. Alwall, R. Frederix, S. Frixione, V. Hirschi, F. Maltoni, O. Mattelaer, H.-S. Shao, T. Stelzer, P. Torrielli, M. Zaro, JHEP 1407, 079 (2014)

67. http://feynrules.irmp.ucl.ac.be/wiki/VLQ-bsingletvl

68. A. Alloul, N.D. Christensen, C. Degrande, C. Duhr, B. Fuks, Comput. Phys. Commun. 185, 2250-2300 (2014)

69. M. Tanabashi et al., Particle Data Group, Phys. Rev. D 98, 030001 (2018)

70. For a comprehensive review, H.J. He, Y.P. Kuang, C.P. Yuan, arXiv:hep-ph/9704276

71. H.J. He, W.B. Kilgore, Phys. Rev. D 55, 1515 (1997)

72. H.J. He, Y.P. Kuang, C.P. Yuan, Phys. Rev. D 51, 6463 (1995)

73. H.J. He, Y.P. Kuang, C.P. Yuan, Phys. Rev. D 55, 3038 (1997)

74. H.J. He, Y.P. Kuang, X. Li, Phys. Rev. Lett. 69, 2619 (1992)

75. T. Sjöstrand, S. Ask, J.R. Christiansen et al., Comput. Phys. Commun. 191, 159 (2015)
76. J. de Favereau et al., DELPHES 3. JHEP 02, 057 (2014)

77. E. Leogrande, P. Roloff, U. Schnoor, M. Weber, arXiv:1909.12728 [hep-ex]

78. M. Boronat, J. Fuster, I. Garcia, E. Ros, M. Vos, Phys. Lett. B 750, 95-99 (2015)

79. M. Boronat, J. Fuster, I. Garcia, P. Roloff, R. Simoniello, M. Vos, Eur. Phys. J. C 78, 144 (2018)

80. E. Conte, B. Fuks, G. Serret, Comput. Phys. Commun. 184, 222256 (2013)

81. E. Conte, B. Dumont, B. Fuks, C. Wymant, Eur. Phys. J. C 74, 3103 (2014)

82. G. Cowan, K. Cranmer, E. Gross, O. Vitells, Eur. Phys. J. C 71, 1554 (2011). (Eur. Phys. J. C 73, 2501 (2013)) 